\title{
An Autopsy Case of Mycobacterium abscessus Pulmonary Infection Complicated with Rheumatoid Arthritis
}

\author{
Kumi Fujita ${ }^{1,2}$, Eisaku Tanaka ${ }^{3}$, Kazuhiro Hatta ${ }^{1}$, Yoshiaki Kori ${ }^{1}$ and Yoshio Taguchi ${ }^{3}$
}

\begin{abstract}
We present the case of a 70-year-old man diagnosed with Mycobacterium abscessus pulmonary infection complicated with rheumatoid arthritis who was treated with corticosteroids. He died despite treatment according to the recommended regimen of imipenem, clarithromycin, and amikacin. Autopsy revealed granulomatous lesions throughout the bilateral lungs. We conclude that $M$. abscessus infection may have a fatal outcome because of the drug resistance of the pathogen. This case suggests that rheumatoid arthritis might be a risk factor for $M$. abscessus pulmonary infection, but further studies are necessary for clarification.
\end{abstract}

Key words: Mycobacterium abscessus, drug resistance, rheumatoid arthritis, corticosteroid

(Inter Med 47: 1273-1276, 2008)

(DOI: 10.2169/internalmedicine.47.1023)

\section{Introduction}

Mycobacterium abscessus is a species of rapidly growing mycobacteria (RGM), and from among RGM, it is the most common pathogen causing pulmonary infection (80\%). Further, it is resistant to conventional antibiotics and antituberculosis agents. Although it is susceptible to imipenem (IPM), clarithromycin (CAM) and amikacin (AMK), no effective drug regimen has been firmly established. The prognosis is poor, and there are few reports of successfully treated cases or autopsy cases of the lethal disease $(1,2)$.

\section{Case Report}

In April 2002, a 70-year-old man was admitted to our hospital because of complaints of fever and productive cough. He had a past history of admission due to bronchopneumonia in August 2001. During the past admission, $M$. abscessus was detected in a sputum culture on one occasion, but the organism was not considered pathogenic. His symptoms and findings from chest scans improved shortly after treatment with conventional antibiotics; hence, he was discharged without further study. Although he occasionally experienced relapsing pyrexia after discharge, we assessed his condition as aspiration pneumonia. He was admitted this time because his symptoms did not improve despite conventional treatment.

The patient had been diagnosed with rheumatoid arthritis (RA) with lung fibrosis (Fig. 1), and oral prednisolone had been administered for 10 years. His medical history included old tuberculosis, emphysema, and peptic ulcer disease from 20 years earlier. He had smoked 30 cigarettes a day for 40 years. He had no family history of any significant disease.

On admission, he was malnourished and appeared chronically ill. His consciousness was drowsy. His height was 160 $\mathrm{cm}$, and body weight was $33.4 \mathrm{~kg}$. Body temperature was $38.5^{\circ} \mathrm{C}$, and blood pressure was $80 / 50 \mathrm{mmHg}$. His heart rate was 100 beats per minute, and respiratory rate was 20 breaths per minute. Coarse crackles and rhonchi were heard over the bilateral lungs. Deformity of the joints was documented, but no lymphadenopathy was observed. Blood examination revealed a white cell count of $18,900 / \mu \mathrm{L}$ and $\mathrm{C}$ reactive protein concentration of $8.3 \mathrm{mg} / \mathrm{dL}$. Blood chemistry showed no remarkable findings, except a rheumatoid factor of $70.5 \mathrm{IU} / \mathrm{mL}$ and a slight elevation in aspartate aminotransferase levels. Arterial blood gas values while breathing ambient air were $\mathrm{pH}, 7.454 ; \mathrm{pCO}_{2}, 33.7 \mathrm{mmHg}$; $\mathrm{pO}_{2}, 79.1 \mathrm{mmHg}$; base excess, $0.9 \mathrm{mmol} / \mathrm{L} ; \mathrm{HCO}_{3}, 23.6$ $\mathrm{mmol} / \mathrm{L}$; and arterial oxygen saturation $\left(\mathrm{SaO}_{2}\right), 96.3 \%$. A

${ }^{1}$ Department of General Medicine, Tenri Hospital, Tenri, ${ }^{2}$ Tenri Institute of Medical Research, Tenri and ${ }^{3}$ Department of Respiratory Medicine, Tenri Hospital, Tenri

Received for publication February 17, 2008; Accepted for publication April 9, 2008

Correspondence to Dr. Kumi Fujita, fujiko@tenriyorozu-hp.or.jp. 


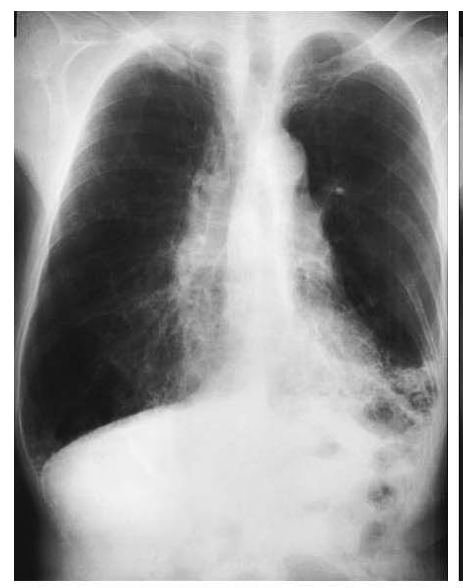

October,2000

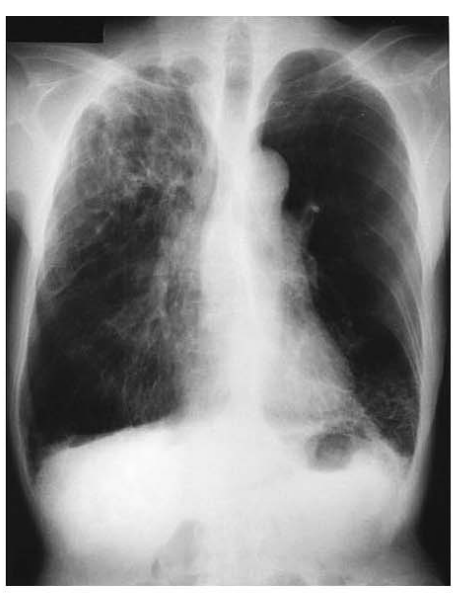

August,2001

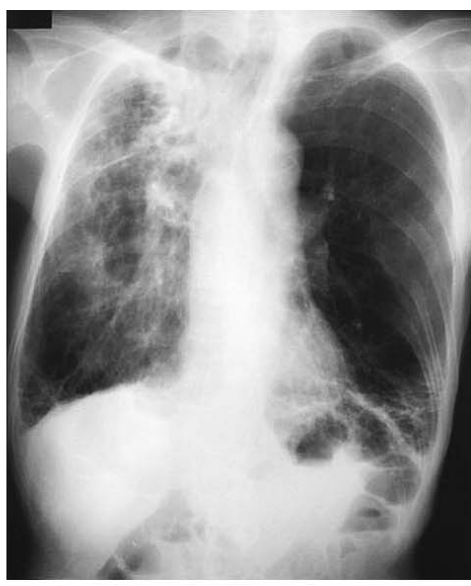

April,2002

Figure 1. Chest radiographs.

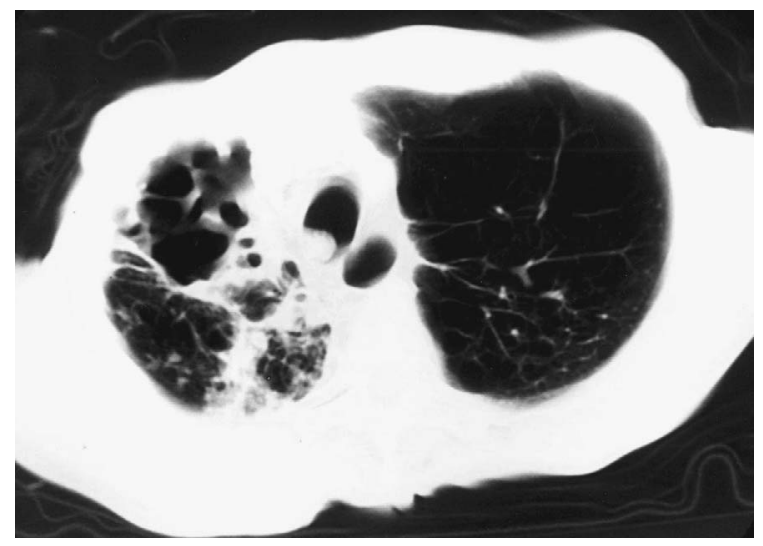

Figure 2. Computed tomography scans of the chest.

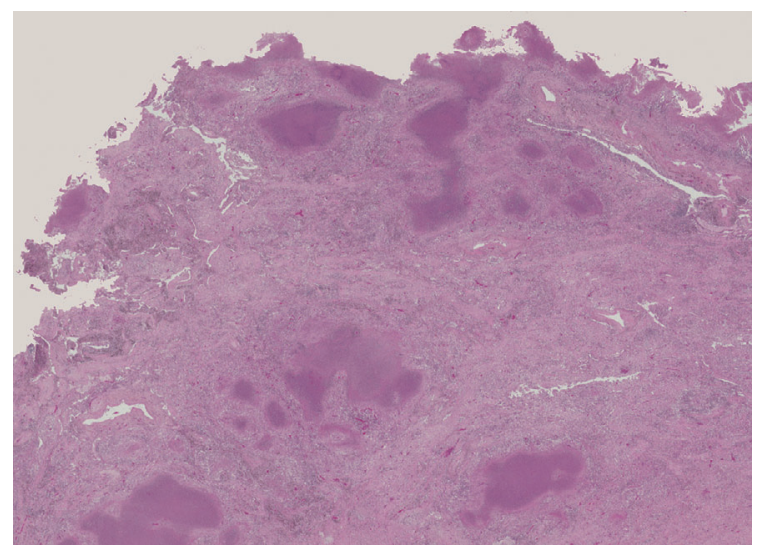

Figure 3. Cavitary lesion in the right upper lung (Microphotograph, Hematoxylin and Eosin staining, $\times 1$ ).

chest radiograph obtained in August 2001 (Fig. 1) revealed a cavitary lesion with surrounding dense consolidation that was not observed in the scan obtained in October 2000. A computed tomography scan of the chest confirmed a huge cavity with a dense wall in the patient's right upper lobe (Fig. 2).

Smears and cultures of the sputum for acid-fast bacilli

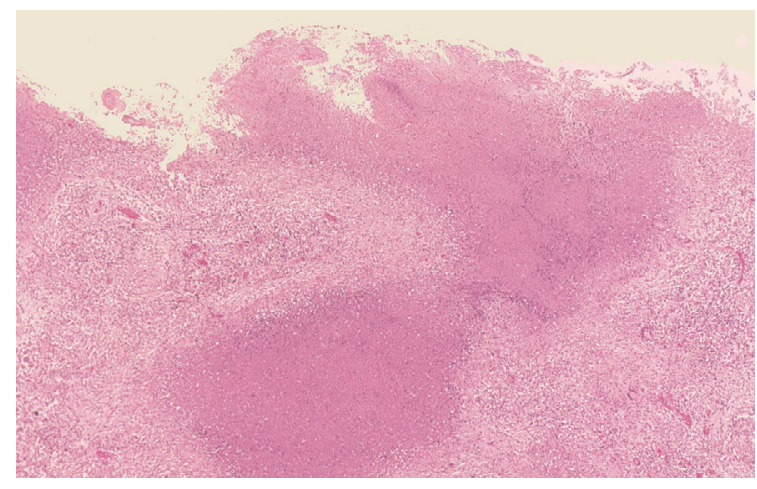

Figure 4. Granuloma in the cavity wall (Microphotograph, Hematoxylin and Eosin staining, $\times 40)$.

yielded positive results on 3 successive occasions. The isolate was identified as $M$. abscessus by DNA-DNA hybridization (DDH Mycobacteria; Kyokuto, Tokyo, Japan) (3). A drug susceptibility test by broth microdilution method (4) revealed the following susceptibilities of the isolated $M$. $a b$ scessus to various drugs: AMK, $<16 \mu \mathrm{g} / \mathrm{mL} ; \mathrm{IPM},<4 \mu \mathrm{g} /$ $\mathrm{mL}$; levofloxacin, $>4 \mu \mathrm{g} / \mathrm{mL}$; ciprofloxacin, $>2 \mu \mathrm{g} / \mathrm{mL}$; and minocycline, $>8 \mu \mathrm{g} / \mathrm{mL}$. A regimen of IPM/CS (30 mg/kg, i. v.), AMK (6 mg/kg, i.v.) and CAM (12 mg/kg, per os) was started. Despite this treatment, the patient's symptoms and findings from chest scans did not show any improvement. Progressive respiratory failure ensued, and the patient died 4 months later.

On autopsy, both upper lungs were occupied by irregular cavities. Multiple granulomas with caseous necrosis were observed all over the bilateral lungs, particularly around the cavities (Figs. 3, 4). Acid-fast bacilli were recognized within the granulomas by Ziehl-Neelsen staining (Fig. 5). Granulomas were also found in the visceral pleura and hilar lymph nodes (Fig. 6). Diffuse fibrosis with cystic changes and pleural thickening were observed mainly in the lower lungs. There were no remarkable findings in other organs. 


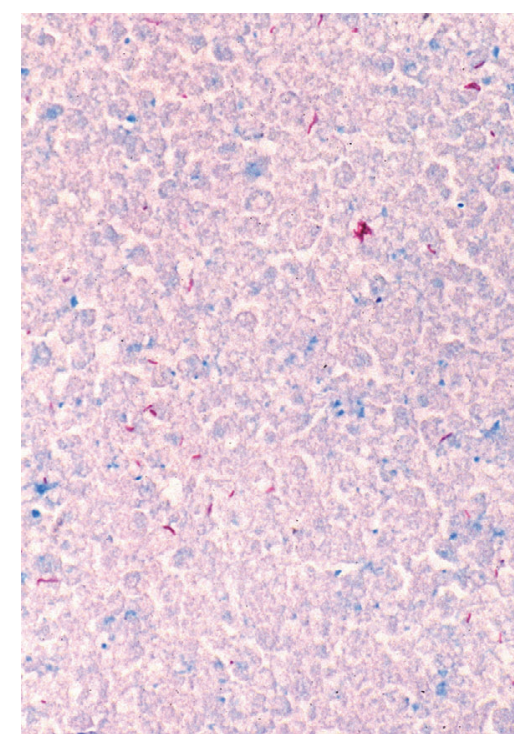

Figure 5. Acid-fast bacilli in the granuloma (Microphotograph, Ziehl-Neelsen, $\times 400$ ).

\section{Discussion}

M. abscessus is a species of rapidly growing mycobacteria (RGM) that belongs to the group of non-tuberculous mycobacterium (NTM) $(1,2)$. It can cause severe pulmonary infection, and there are few successfully treated case reports in the literature because of the multi-drug resistance of the bacteria (5-9). Griffith et al discussed the details of RGM pulmonary infection and reported that the prognosis of $M$. abscessus pulmonary infection is poor and that the mortality rate is $20 \%$ (2). Relapse after remission is frequent, and successful treatment of $M$. abscessus pulmonary disease is considered difficult. In the present case, M. abscessus was first detected in the sputum in August 2001, but the diagnostic criteria established by the American Thoracic Society in 1997 were not satisfied because the criteria required 2 or more cultures that were positive for the pathogen (10). Before the patient was diagnosed with $M$. abscessus infection, he had experienced relapsing pyrexia. His condition improved with conventional antibiotic therapy, and specific treatment was not started. That delay in diagnosis may have caused the disease to worsen. Although we treated him with the recommended regimen of CAM, IPM/CS and AMK for more than 4 months after diagnosis, the patient died. It is not clear why these drugs were not effective despite the susceptibility of the pathogen to these drugs in an in vitro drug susceptibility test.

Autopsy revealed multiple necrotizing granulomatous lesions with acid-fast bacilli over almost the entire area of both lungs. This finding implies that the disease remained severe, regardless of the long-term treatment. The huge cavitary lesion on the right upper lobe was not observed on chest radiographs until 1 year previously. The wall of the cavity comprised necrotizing granulomatous lesions. Active granulomatous changes caused destruction of the structure

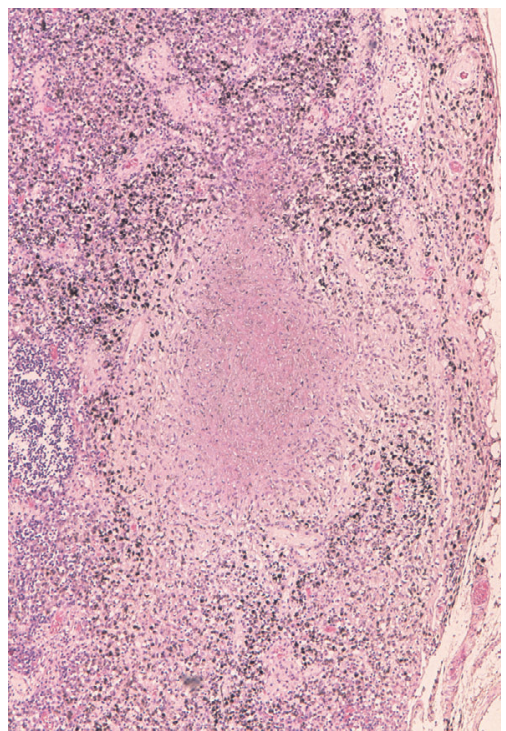

Figure 6. Granuloma in the hilar lymph node (Microphotograph, Hematoxylin and Eosin staining, $\times 40$ ).

of the patient's upper lungs and cavity formation for approximately 1 year. Fibrous changes, probably due to RA, were observed around the bilateral lower lungs. It is unclear if these changes predisposed our patient to NTM infection.

The present patient had a history of RA treated with corticosteroids, gastric ulcer, and underlying pulmonary lesions. These are all regarded as predisposing factors of NTM pulmonary diseases. Griffith et al regarded gastro-oesophageal disorders with chronic aspiration and old tuberculosis, but not treatment with RA, corticosteroid, or immunosuppressive agents, as risk factors of RGM pulmonary infection (2). Dreisin et al identified a relationship between RGM pulmonary disease and RA (9); however, other reports support that RA is a risk factor of NTM infection. RA patients tend to be immunosuppressed because of the underlying disease and its treatment. Further, pulmonary lesions due to RA would complicate the pathology of NTM pulmonary diseases. Origuchi et al reported 2 cases of Mycobacterium avium pulmonary infection associated with RA (11). They suggested that RA would be associated with NTM pulmonary diseases even in the absence of treatment with immunosuppressant agents. Watkin et al reported a case of Mycobacterium kansasii pulmonary disease with RA and discussed whether the suppression of cellular immunity or immunosuppression mediated by neutrophils would increase the tendency of infection in RA patients (12). In recent times, more aggressive treatment of RA with biological drugs is recommended. The risk of NTM pulmonary infection among RA patients would increase under such a condition that had not been previously experienced (13), and we should be cautious of opportunistic infections, including NTM.

In the present case, granulomatous lesions were found not only in the lungs but also in the hilar lymph nodes and visceral pleura. The lesions of tuberculosis are said to spread through 3 courses: 1) endobronchial spread, 2) contiguous spread, and 3) lymphohematogenous spread (14). In cases of 
primary tuberculosis, lesions of the mediastinal or hilar lymph nodes are frequently observed. In secondary tuberculosis (affecting adults), the lesions tend to localize within the lungs. There are no case reports of NTM pulmonary diseases with lesions infiltrating into the mediastinal or hilar lymph nodes, except cases of acquired immunodeficiency syndrome (AIDS) patients. The present case might suggest the possibility of extrapulmonary spread of NTM infection in patients other than AIDS patients.
We present an autopsy case of $M$. abscessus pulmonary infection associated with RA. The patient died despite treatment with the standard regimen to which the pathogen was susceptible in an in vitro drug susceptibility test. RA is suggested as a risk factor of NTM pulmonary infection.

A summary of this paper was presented at the 169th meeting of the Kinki branch of the Japanese Society of Internal Medicine, December 14, 2002, Osaka, Japan.

\section{References}

1. American Thoracic Society. An official ATS/IDSA statement: Diagnosis, treatment, and prevention of nontuberculous mycobacterial diseases. Am J Respir Crit Care Med 175: 367-416, 2007.

2. Griffith DE, Girard WM, Wallace RJ. Clinical features of pulmonary disease caused by rapidly growing mycobacteria. An analysis of 154 patients. Am Rev Respir Dis 147: 1271-1278, 1993.

3. Ezaki T. Rapid genetic identification system of mycobacteria. Kekkaku 67: 803-808, 1992 (in Japanese, Abstract in English).

4. Brown BA, Swenson JM, Wallace RJ Jr. Broth microdilution test for rapidly growing mycobacteria. In: Clinical Microbiology Procedures Handbook. vol. 1. Isenberg HD, Ed. American Society for Microbiology, Washington, D.C., 1992: 5.11.1-5.11.10.

5. Tanaka E, Kimoto T, Tsuyuguchi K, Suzuki K, Amitani R. Successful treatment with faropenem and clarithromycin of pulmonary Mycobacterium abscessus infection. J Infect Chemother 8: 252$255,2002$.

6. Takemura Y, Iwasaki Y, Minagawa T, et al. Mycobacterium abscessus infection complicated with diabetes mellitus. Nihon Kokyuki Gakkai Zasshi 40: 61-65, 2002 (in Japanese, Abstract in English).

7. Tagawa A, Ikehara $\mathrm{K}$, Nishiyama $\mathrm{H}$, et al. A case of lung infection due to Mycobacterium abscessus. Nihon Kokyuki Gakkai Zasshi 41: 546-550, 2003 (in Japanese, Abstract in English).

8. Shikama Y, Kamio Y, Kuriu K, et al. A case of Mycobacterium abscessus pulmonary infection; effectiveness of clarithromycin, amykacin and imipenem/cilastatin. Nihon Kokyuki Gakkai Zasshi 44: 800-806, 2006 (in Japanese, Abstract in English).

9. Dreisin RB, Scoggin C, Davidson PT. The pathogenicity of Mycobacterium fortuitum and Mycobacterium chelonei in man: a report of seven cases. Tubercle 57: 49-57, 1976.

10. American Thoracic Society. Diagnosis and Treatment of Disease Caused by Nontuberculous Mycobacteria. Am J Respir Crit Care Med 156: S1-S25, 1997.

11. Origuchi $T$, Migita $K$, Kawakami A, et al. Atypical mycobacteriosis in two patients with rheumatoid arthritis. Mod Rheumatol 12: 76-79, 2002.

12. Watkin SW, Bucknall RC, Nisar M, Agnew RAL. Atypical mycobacterial infection of the lung in rheumatoid arthritis. Ann Rheum Dis 48: 336-338, 1989.

13. Thomas JE, Taoka CR, Gibbs BT, Fraser SL. Fatal pulmonary Mycobacterium abscessus infection in a patient using etanercept. Hawaii Med J 65: 12-15, 2006.

14. Procop GW, Tazelaar HD. Tuberculosis and other mycobacterial infections of the lung. In: Thurlbeck's Pathology of the Lung. 3rd ed. Churg AM, Myers JL, Tazelaar HD, Wright JL, Eds. Thieme, New York, 2005: 219-248.

\section{(C) 2008 The Japanese Society of Internal Medicine http://www.naika.or.jp/imindex.html}

